\title{
AN ANALYTICAL METHOD TO RND WORKSPACE OF A ROBOTIC MANIPULATOR
}

\author{
Khushdeep Goyal ${ }^{1 *}$ and Davinder Sethi ${ }^{2}$ \\ ${ }^{1}$ Yadavindra College of Engineering, Punjabi University Guru Kashi Campus, TALWANDI SABO-151302, \\ INDIA \\ ${ }^{2}$ Baba Hira Singh Bhattal College of Engineering \& Technology, LehraGaga, INDIA \\ *Corresponding e-mail: khushgoyal@yahoo.com
}

\begin{abstract}
The workspace of a Robot is determined by an analytical method. The method is applicable to kinematic chains that can be modeled using the Denavit-Hartenberg representation for serial kinematic chains. This method is based upon analytical criteria for determining singular behavior of the mechanism. By manipulating the Jacobian of the robot by the row rank deficiency condition, the singularities are computed. Then these singularities are substituted into the constraint equations to parameterize singular surfaces. The boundary conditions of the joints are substituted to obtain the other set of singularities. These singularities are substituted in the wrist vector to obtain the range of motion of the robot wrist in three dimensional space, which is the workspace of the Robot. These singularities are plotted in Matlab to develop all the surfaces enveloping the workspace of the Robot. The priactical examples of RV-M1 MITSIBUSHI ROBOT and 3 DOF spatial manipulator are treated with this method.
\end{abstract}

Key Words: Jacobian, Workspace, Singularities, Degree of freedom

\section{INTRODUCTION}

Numerical methods for determining the workspace boundaries of serial manipulators have been developed in recent years. Exact computation of the workspace and its boundary is of significant importance because of its impact on manipulator design, manipulator placement in an environment, and manipulator dexterity. Ricard and Gosselin ${ }^{1}$ explained one method to determine the Workspace of complex planar Robotic Manipulators. In this paper the authors gave a method which is based on the use of joint limits to obtain equations describing the limiting curves. These limiting curves are then segmented at their mutual intersections and validated. The resulting set of curves from the envelope of the workspace.
Ceccarelli $^{2}$ presented a synthesis algorithm for three revolute manipulators by using an algebraic formulation of workspace boundary and explained a synthesis algorithm for general three degree of freedom manipulator. The synthesis equations are formulated as a set of nonlinear algebraic equations whose unknowns are workspace structural coefficients. The authors Snyman and Plessis ${ }^{3}$ gave an optimization approach to the determination of the boundaries of manipulator workspace.

This numerical method consists of finding a suitable radiating point in the output coordinate space and then determining the points of intersection of a representative pencil of rays, emanating from the radiating point, with the boundary of the accessible set.

\section{Nomenclature}

$\begin{array}{ll}\mathrm{q}_{\mathrm{i}} & \text { Joint Variables } \\ \theta_{i}, \mathrm{\alpha}_{\mathrm{i}} & \text { Angles in degrees } \\ { }^{\mathrm{i}-1} \mathrm{~T}_{\mathrm{i}} & \text { Transformation Matrix } \\ { }^{0} \mathrm{R}_{1} & \text { Rotation Matrix } \\ { }^{0} \mathrm{P}_{\mathrm{i}} & \text { Position Vector } \\ \Phi(\mathrm{q}) & \text { Constraint Equation } \\ \hat{\mathrm{J}}(\mathrm{q}) & \text { Jacobian of Position Vector } \\ \Psi^{\mathrm{i}}\left(\mu_{\mathrm{i}}\right) & \text { Set of Surfaces }\end{array}$

J oumal of Mechanical Engineering, Vol. ME 41, No. 1, J une 2010 Transaction of the Mech. Eng. Div., The Institution of Engineers, Bangladesh 
Yang et $\mathrm{al}^{4}$ have described that placement of an open loop robotic manipulator in a working environment is characterized by defining the position and orientation of the manipulator's base with respect to a fixed reference frame.

Kumar and Waldron ${ }^{5}$ presented another algorithm to compute the manipulator's workspace. $\mathrm{Bi}$ and $\mathrm{Lang}^{6}$ have proposed a forward kinematic model for determining the workspace of tripod machine tool. The joint motions are used to calculate the workspace. Malek and $\mathrm{Yeh}^{7}$ explained a broadly applicable formulation for representing the boundary of swept geometric entities using Jacobian rank deficiency conditions. A constraint function is defined as one entity is swept along another. Boundaries in terms of inequality constraints imposed on each entity are considered which gives rise to an ability of modeling complex solids. The intersection curves between two parametric surfaces are determined by the method explained by Malek and $\mathrm{Yeh}^{8}$. This paper presents a method for determining the intersection curves of two intersecting parametric surfaces using continuation methods. Cao et $\mathrm{al}^{9}$ used a numerical method based on random probatility to generate the planar boundary curves of spatial robot in its main working plane. Then 3D shape and volume of robot workspace are generated by commercial software unigraphics. Determination of workspace boundaries is also referred by Jo et $\mathrm{al}^{10}$. Pitarch et $\mathrm{al}^{11}$, Malek ${ }^{12}$, Tsai and Soni ${ }^{13}$, Sheen and Luo ${ }^{14}$. Malek and Yang $^{15}$ demonstrated a mathematical formulation for creation of solid models. It is shown that Denavit-Hartenberg representation method adopted from kinematics is well suited for the representation of soid models that are created as a result of multiple sweeps.

\section{METHODOLOGY}

In order to analytically find expressions for the boundary surfaces of manipulators, it is necessary

1. To develop a set of analytical criteria to obtain the positioning of the wrist in terms of generalized coordinates.

2. Determine the boundary surfaces due to singularities associated with the set, and

3. Determine the subset of these surfaces due to joint limits. Combine all the surfaces to develop the workspace.

The mathematics to determine above is explained in the following sections.

\section{Finding wrist accessible position vector}

The first step is to determine the wrist accessible position vector of the robot. For determining the wrist accessible output set the property that, two coordinate systems is orthogonal to the z-axis of the other system are related by a Euclidean motion depending on four parameters, is used. Consider two coordinate frames in space, the first denoted by $\mathrm{x}_{0}, \mathrm{y}_{0}, \mathrm{z}_{0}$ with origin at $\mathrm{O}_{0}$ and the second by $\mathrm{x}_{1}, \mathrm{y}_{1}$, $\mathrm{z}_{1}$ with origin at $\mathrm{O}_{1}$. The point $\mathrm{P}$ is an arbitrary point in space. The vector ${ }^{0} \mathrm{v}$ is the vector coordinates of $\mathrm{P}$ with respect to $\mathrm{x}_{0}, \mathrm{y}_{0}, \mathrm{z}_{0}$ reference frame. ${ }^{1} \mathrm{~V}$ is the vector of coordinates of $\mathrm{P}$ with respect to frame $1 .{ }^{0} b_{1}$ is the vector describing the origin of frame 1 with respect to frame 0 as shown in figure 1.

Using geometry, the position of point $\mathrm{P}$ with respect to frame 0 can be expressed as:

$$
{ }^{0} \mathrm{v}={ }^{0} \mathrm{~b}_{1}+{ }^{0} \mathrm{R}_{1}{ }^{1} \mathrm{v}
$$

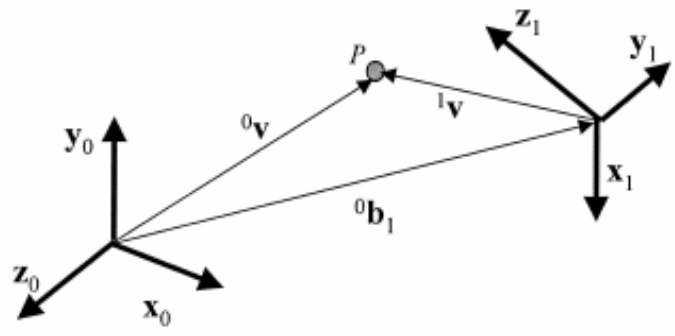

Figure..1 Representation of a point $\mathrm{P}$

Where ${ }^{0} \mathrm{R}_{1}=\left[\begin{array}{lll}\mathrm{x}_{1} & \mathrm{y}_{1} & \mathrm{z}_{1}\end{array}\right]$ is the rotation matrix relating the orientation of both frames. In order to achieve a compact representation and to simplify the mathematics between subsequent coordinate frames, the homogeneous representation of a generic vector $\mathrm{v}$ can be introduced as the vector $\mathrm{v}^{*}=\left[\begin{array}{ll}\mathrm{v}^{\mathrm{T}} & 1\end{array}\right]^{\mathrm{T}}$ formed by adding a fourth unit component. The coordinate transformation can be written as

$$
{ }^{0} \mathrm{~T}_{1}=\left[\begin{array}{cc}
{ }_{0} R_{1} & { }_{0} b_{1} \\
0 & 1
\end{array}\right]
$$

Hence, transforming a vector from one coordinate frame to another is written as

$$
{ }^{0} \mathrm{~V} *={ }^{0} \mathrm{~T}_{1}{ }^{1} \mathrm{~V} *
$$

In order to generate the matrix relating any two transformations, a minimal representation of only four parameters is necessary to describe one coordinate system with respect to the other. These four parameters are called Denavit-Hartenberg (D$\mathrm{H})$ parameters. The D-H representation provides a systematic method for describing the relationship between adjacent links. The $4 \times 4$ transformation matrix describing a transformation from link (i-1) to link i for a revolute joint is :

$$
{ }^{\mathrm{i}-1} \mathrm{~T}_{\mathrm{i}}=\left[\begin{array}{cccc}
\cos \theta & -\cos \alpha_{i} \sin \theta & \sin \alpha_{i} \sin \theta & a_{i} \cos \theta \\
\sin \theta & \cos \alpha_{i} \sin \theta & -\sin \alpha_{i} \cos \theta & a_{i} \sin \theta \\
0 & \sin \alpha_{i} & \cos \alpha_{i} & d_{i} \\
0 & 0 & 0 & 1
\end{array}\right]
$$




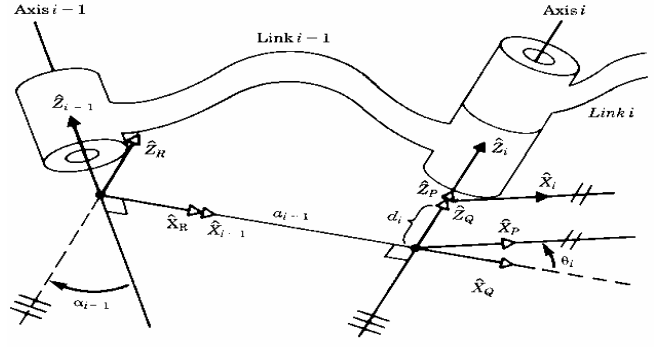

Figur.2. Link coordinates system and its Parameters

where $\theta_{i}$, depicted in Fig. 2.2, is the joint angle from $\mathrm{x}_{\mathrm{i}-1}$ to the $\mathrm{x}_{\mathrm{i}}$ axis, $d_{i}$ is the distance from the origin of the (i-1)th coordinate frame to the intersection of the $\mathrm{z}_{\mathrm{i}-1}$ axis with the $\mathrm{x}_{\mathrm{i}}, a_{i}$ is the offset distance from the intersection of the $\mathrm{z}_{\mathrm{i}-1}$ axis with the $x_{i}$ axis, and $\alpha_{i}$ is the offset angle from the $\mathrm{z}_{\mathrm{i}-1}$ axis to the $\mathrm{z}_{\mathrm{i}}$ axis.

The homogeneous transformation matrix ${ }^{0} \mathrm{~T}_{\mathrm{i}}$ that specifies the configuration of the $i$ th frame with respect to the base coordinate system is the product of successive transformation matrices of ${ }^{\mathrm{i}-1} \mathrm{~T}_{\mathrm{i}}$,

$$
\begin{gathered}
{ }^{0} \mathrm{~T}_{\mathrm{i}}={ }^{0} \mathrm{~T}_{1 .}{ }^{1} \mathrm{~T}_{2} \ldots .{ }^{\mathrm{i}-1} \mathrm{~T}_{\mathrm{i}}=\prod_{j=1}^{i}{ }^{\mathrm{j}-1} \mathrm{~T}_{\mathrm{j}} \\
{ }^{\mathrm{i}-1} \mathrm{~T}_{\mathrm{i}}=\left[\begin{array}{cc}
{ }_{0} R_{i} & 0 P_{i} \\
0 & 1
\end{array}\right]
\end{gathered}
$$

where ${ }^{0} \mathrm{R}_{\mathrm{i}}$ is the rotational matrix from the $\mathrm{i}-1$ coordinate frame to $0^{\text {th }}$ coordinate frame. And ${ }^{0} \mathrm{P}_{\mathrm{i}}$ is the position vector with respect to the $0^{\text {th }}$ coordinate frame. This is also called wrist accessible position vector when $\mathrm{i}=$ number of degrees of freedom of the robot.

In order to determine the boundary of the wristaccessible output set for a mechanism, the singularities (both internal and external) can be computed by proper manipulation of the Jacobian of the mechanism. The first order and second order singularities are computed in this thesis work. These singularities are substituted in the wrist vector to get the range of motion of the wrist.

For a given configuration of the manipulator, the generalized coordinates satisfy independent holonomic kinematic constraint equations of the form:-

$$
\Phi(q)=0
$$

Where $\Phi: R_{n} \rightarrow R^{1}$ is a smooth function, and 1 is the number of constraint equations. In addition, the generalized coordinates $\mathrm{q}$ are subject to inequality constraints representing limits.

$$
\mathrm{q}_{\mathrm{i}}{ }^{\mathrm{min}} \leq \mathrm{q}_{\mathrm{i}} \leq \mathrm{q}_{\mathrm{i}}^{\max } \quad, \mathrm{i}=1,2,3, \ldots, \mathrm{n}
$$

To impose joint limits of the actuators in terms of the generalized variables, it is possible to transform a constraint of the form as equation 2.14 into an equation by introducing a new generalized coordinate $\lambda_{\mathrm{i}}$, such that the inequality constraint of equation 2 can be written as:

$$
\mathrm{q}_{\mathrm{i}}=\mathrm{a}_{\mathrm{i}}+\mathrm{b}_{\mathrm{i}} \sin \lambda_{\mathrm{i}}
$$

where $\mathrm{a}_{\mathrm{i}}=\left(\mathrm{q}_{\mathrm{i}}{ }^{\mathrm{max}}+\mathrm{q}_{\mathrm{i}}{ }^{\mathrm{min}}\right) / 2$ and $\mathrm{b}_{\mathrm{i}}=\left(\mathrm{q}_{\mathrm{i}}^{\max }-\mathrm{q}_{\mathrm{i}}^{\mathrm{min}}\right) / 2$ are midpoint and half-range of the inequality constraint.

\section{Jacobian of the wrist accessible output set}

The first order singularities are computed by equating the determinant of the Jacobian of the mechanism to zero and then computing the roots. The constraint Jacobian of the constraint function $\Phi(q)$ for a certain configuration can be calculated as

$\hat{\mathrm{J}}(\mathrm{q})=\left[\frac{\partial \Phi(q)}{\partial q_{i}}\right]$

The above equation is called the Jacobian of the position vector.

The Jacobian has dimension $n \times 3$. Where $n$ is the number of independent variables, which may be a square or non square matrix. The method of computing Jacobian singularities is generalized to computing sets of singular parameters. This formulation generalizes the Jacobian method to the computation of the boundary of any manipulator with any number of independent variables i.e. degrees of freedom. The Jacobian method is extended to a row rank deficiency method, i.e. the possibility of handling a non square Jacobian. In this general formulation, the resulting Jacobian is not square and there are three types of singular behavior observed. The term singular behavior is given to geometric entities that exist in the accessible set that cause difficulties in satisfying equation 10. For a singular entity inside the accessible set, it is not possible to cross the entity in a direction normal to its surface.

If the Jacobian is square matrix then the singularities are computed by putting the determinant of the Jacobian equal to zero such that

$$
\left|\Phi_{q}\right|=0
$$

$$
\text { Where } \Phi_{\mathrm{q}}=\left[\frac{\partial \Phi(q)}{\partial q_{i}}\right]
$$

From equation (10) and solving the resulting equation in terms of the independent variables. The solution gives first set of singularities.

\section{Rank-deficiency singularity set}

In general, the Jacobian matrix is not square. Therefore, a rank-deficiency criterion is developed. Consider the $3 \times n$ matrix $\hat{J}^{T}(q)$ over $R^{n}$. If $\operatorname{rank}(\hat{J})$ $=r$, then $\hat{\mathrm{J}}$ has $r$ linearly independent rows. Let $\hat{J}_{1}$ be an $3 \times \mathrm{r}$ sub Jacobian of $\hat{\mathbf{J}}$ whose columns are precisely the $r$ linearly independent columns of $\hat{\mathbf{J}}$

J oumal of Mechanical Engineening, Vol. ME 41, No. 1, J une 2010 Transaction of the Mech. Eng. Div., The Institution of Eng ineers, Bangladesh 
mentioned above. The rank of $\hat{J}_{1}$ is also r. Now let $\hat{\mathbf{J}}_{2}$ be an $\mathrm{r} \times \mathbf{r}$ sub Jacobian of $\hat{\mathrm{J}}$ whose columns are linearly independent. Thus, for $\hat{J}_{2}$ the determinant $\operatorname{det}\left(\hat{\mathrm{J}}_{2}\right) \neq 0$.

The singular behavior occurs for the set of singularities that satisfies:

$$
\mathrm{S}_{1}=\left\{\mathrm{p} € \mathrm{R}^{\mathrm{n}} ; \operatorname{dim} \operatorname{Null}(\hat{\mathrm{J}}(\mathrm{q})) \geq 1, \text { for some } \mathrm{q}\right\}
$$

Where $p$ is a set of constant generalized coordinates (subset of $\mathrm{q}$ ) that renders the analytic Jacobian singular and Null is the nullspace of the matrix $\hat{J}$, i.e. the rows that are dependent. Substituting the set $\mathrm{p} € \mathrm{~S}_{1}$ into wrist vector yields a hyperentity. The hyperentities are geometrical entities which appear in the boundary. These hyperentities may be on the boundary or inside it. The set $S_{1}$ can be determined by setting the determinants of the sub Jacobians $\left(\hat{J}_{1}, \hat{J}_{2}, \ldots \ldots.\right)$ of $\hat{\mathbf{J}}$ to zero and seeking simultaneous solutions.

Rank-deficiency of reduced-order accessible set At a boundary of one of the surfaces (a parametric limit is encountered), the edge of a geometrical entity is swept. To determine those entities, it is necessary to reduce the order of the accessible set by substituting a value for one of the parameters. Both upper limits and lower limits are considered. Once a reduced set is obtained, the null space criteria of equation 2.10 is applied to reduced set.

Each variable in the set is constrained by an upper and lower limit in terms of inequality constraints such as equation 2.8. Substituting a limit $\left(\mathrm{q}_{i}{ }^{\min }\right.$ or $\mathrm{q}_{\mathrm{i}}^{\mathrm{max}}$ ) into wrist point yields the position vector for the reduced order set (i.e. the set has lost one degree of freedom by fixing one link).

In this case, the Jacobian of $\Phi^{*}\left(\mathrm{q}^{*}\right)=\Phi\left(\mathrm{q}^{*}, \mathrm{q}_{\mathrm{i}}{ }^{0}\right)$, where $\mathrm{q}_{\mathrm{i}}{ }^{0}$ is the limit of the parameter $\mathrm{i}$, and $\mathrm{q}^{*}=\left[\mathrm{q}_{1}, \mathrm{q}_{2}, \ldots \ldots \ldots\right]^{\mathrm{T}}$ is the vector of reduced set, can be defined as:

$$
\hat{\mathrm{J}}^{\mathrm{R}}\left(\mathrm{q}^{*}\right)=\left[\frac{\partial \Phi\left(q^{*}\right)}{\partial q^{*}}\right]
$$

Define a second singularity set analogous to equation 12 as:

$$
\mathrm{S}_{2}=\left\{\mathrm{p} € \mathrm{R}^{\mathrm{n}} ; \operatorname{dim} \operatorname{Null}\left(\hat{\mathrm{J}}\left(\mathrm{q}^{*}\right)\right) \geq 1, \text { for some } \mathrm{q}^{*}\right\}
$$

The set $\mathrm{S}_{2}$ can be determined by equating the determinants of the sub Jacobians of $\left(\hat{\mathrm{J}}\left(\mathrm{q}^{*}\right)\right)$ to zero and simultaneously seeking a solution.

\section{Constraint singularity set}

A boundary is reached when the number of constant parameters equals the dimensions of ${ }^{n} \mathrm{X}_{\mathrm{w}}$ or ${ }^{0} \mathrm{P}_{\mathrm{n}}$.

Entities that are due to active parameter constraints are called constraint singularity sets and are defined by:
$\mathrm{S}_{3}=\left\{\mathrm{p} € \mathrm{R}^{\mathrm{n}} ;\left[\mathrm{q}_{\mathrm{i}}{ }^{0}, \mathrm{q}_{\mathrm{j}}{ }^{0}\right]\right.$, for $\mathrm{i}, \mathrm{j}=1$ to $\left.\mathrm{n} ; \mathrm{i} \neq \mathrm{j}\right\}$

Where $\mathrm{q}_{\mathrm{i}}^{0}$ and $\mathrm{q}_{\mathrm{j}}{ }^{0}$ are the limits of equation 2.8. The constraint singularity set is a combination of all constant parameters, i.e. for each combination of the limits of the constraints, there exists a hyperentity.

\section{Total singularity set}

The total singularity set $\mathrm{S}$ generated by the above criteria is :

$$
\mathrm{S}=\mathrm{S}_{1} \mathrm{US}_{2} \mathrm{US}_{3}
$$

$\mathrm{S}_{1}, \mathrm{~S}_{2}$, and $\mathrm{S}_{3}$ are singularities given by equations 13,14 , and 15 .

It may be possible that singularities generated by one set may be identical to others generated by another set.

\section{Workspace boundary}

Substituting each singularity into the accessible output set, a set of surfaces $\Psi^{1}\left(\mu_{\mathrm{i}}\right)$ are parameterized such that

$\Psi^{\mathrm{i}}\left(\mu_{\mathrm{i}}\right)=\left[\Psi^{1}\left(\mu_{\mathrm{i}}\right), \Psi^{2}\left(\mu_{2}\right), \ldots \ldots, \Psi^{\mathrm{m}}\left(\mu_{\mathrm{m}}\right)\right]$

Where $\mathrm{i}=1, \ldots, \mathrm{m}$. These surfaces determine the boundary of the workspace

\section{EXPERIMENTAL RESULTS Generating workspace of rv-m1 mitsibushi robot}

Fig 3. shows the joints and dimensions of RV-M1 Mitsibushi robotic manipulator. The $\mathrm{DH}$ parameters are given in table 3.1 .

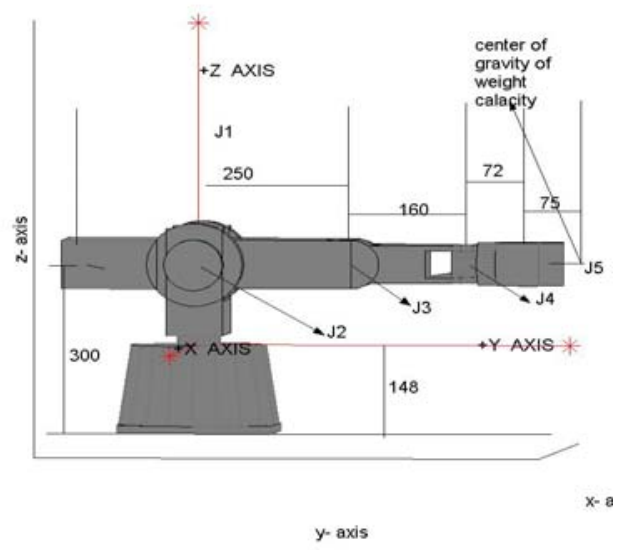

Figure.3.: Mitsubishi RV-M1 Robot joints and dimensions

\section{D-H PARAMETERS}

The D-H parameters for RV-M1 robot manipulator are given in the following table.

J oumal of Mechanical Engineening, Vol. ME 41, No. 1, J une 2010 Transaction of the Mech. Eng. Div., The Institution of Eng ineers, Bangladesh 
Table1: D-H parameters for RV-M1

\begin{tabular}{|l|l|l|l|l}
\hline Joint & $\theta_{i}$ & $\mathrm{~d}_{\mathrm{i}}$ & $\alpha_{\mathrm{i}}$ & $\mathrm{a}_{\mathrm{i}}$ \\
\hline 1 & $\mathrm{q}_{1}$ & 152 & $90^{0}$ & 0 \\
\hline 2 & $\mathrm{q}_{2}$ & 0 & 0 & 250 \\
\hline 3 & $\mathrm{q}_{3}$ & 0 & 0 & 160 \\
\hline 4 & $\mathrm{q}_{4}$ & 0 & 0 & 72 \\
\hline
\end{tabular}

Where the joint limits are:

$$
\begin{aligned}
-150^{0} \leq \mathrm{q}_{1} & \leq 150^{0} \\
-30^{\circ} \leq \mathrm{q}_{2} \leq 100^{\circ} & \leq 110^{0} \leq \mathrm{q}_{3} \leq 0^{\circ} \\
-110^{\circ} & \leq \mathrm{q}_{4} \leq 90^{\circ}
\end{aligned}
$$

After applying above described method, The first singularity set for the RV-M1 robot is: $\mathrm{S}_{1}=\left[\left(\mathrm{q}_{3}=0, \mathrm{q}_{4}=0\right)\right]$ and this singularity plot is shown in Fig. 4 .
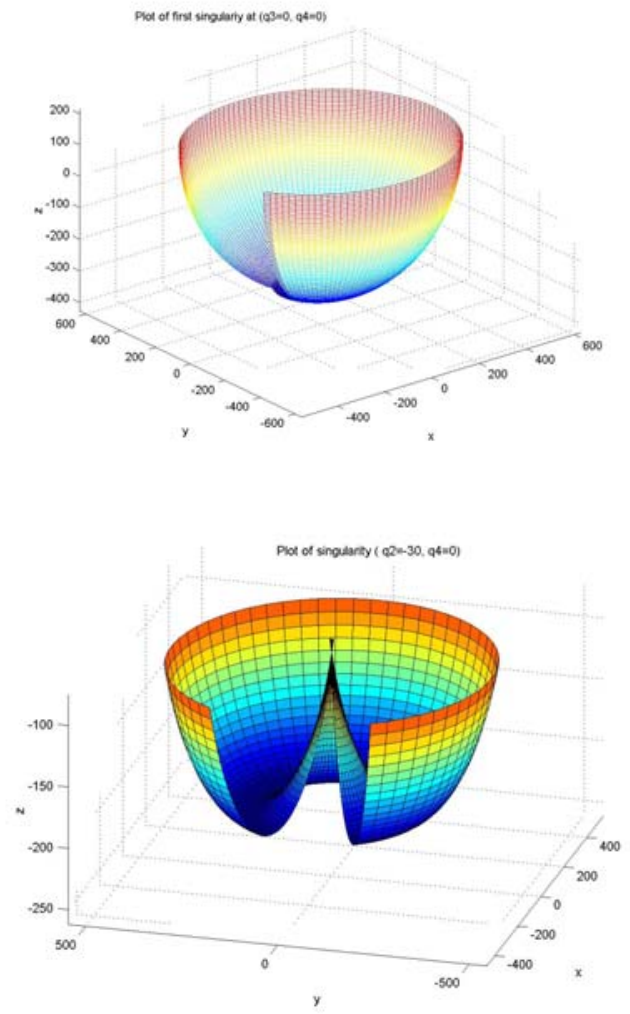

Figure.4 Singularity plot at $\left(\mathrm{q}_{2}=-30, \mathrm{q}_{4}=0\right)$

Similarly all other singularities are generated and are plotted in MATLAB to get all the singular surfaces. And finally all these singularities are combined to obtain the workspace of RV-M1. which is shown in Fig. 5

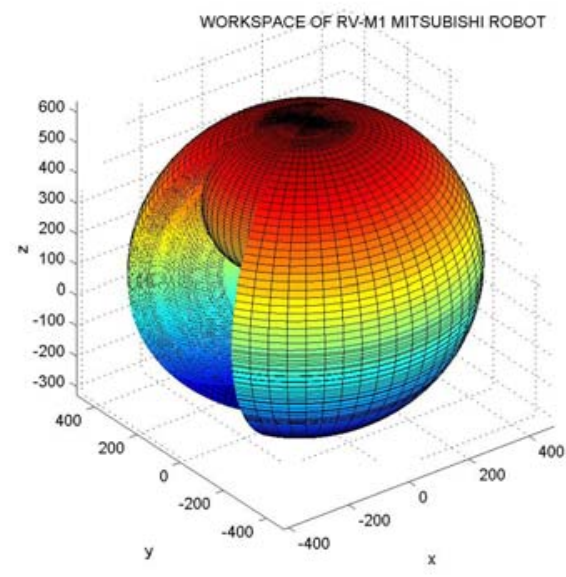

Figure.5 Three dimensional view of workspace RV-M1 Mitsubishi robot

\section{Workspace of 3dof spatial manipulator}

Fig. 6 shows a 3 dof manipulator.

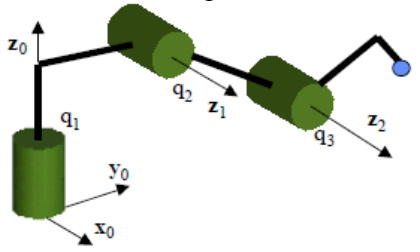

Fig.6 A 3dof manipulator

The application of method described above to this manipulator generates a set of 7 singularities. Combining all the singular surfaces generates the three dimensional workspace, which is shown in fig. 7.

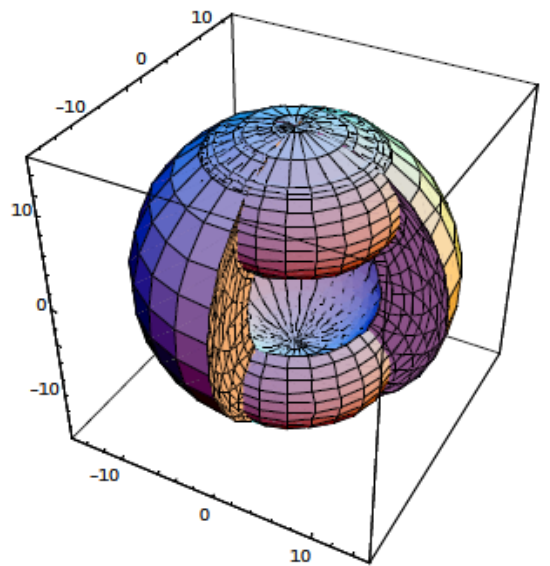

Fig. 7 Workspace of 3dof manipulator

\section{Other examples}

The method explained in this paper is used to determine the workspace of other configuration of manipulators. The fig. 8 (a) and (b) shows the 
workspaces of other combinations of revolute and prismatic joints.
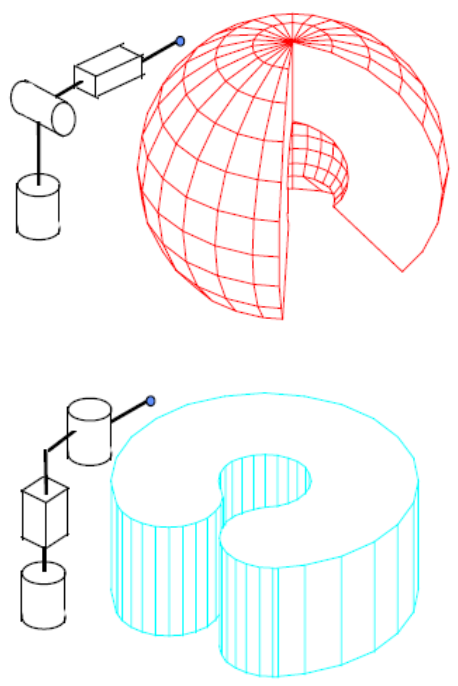

Fig 8 (a) Workspace of RRP and (b) Workspace of RPR configuration

\section{CONCLUSIONS}

An analytical formulation for determining the robot workspace is presented. This formulation is used to develop the Matlab tool box. The workspace constraint function was formulated in terms of generalized coordinates including, inequality constraints imposed on each joint. It was shown that Jacobian rank-deficiency conditions usually applied in robotics analysis to determine degenerate conditions are employed here to generate singular sets. It was shown that three types of singularities may be obtained. First and second types are due to rank-deficiency of the constraint Jacobian matrix and of the reduced-order Jacobian matrix. The third type of singular behavior is due to active parametric constraints. Then the Matlab code is developed to plot all the calculated singularities, which generates the workspace of the Robot. This method is applied to the RV-M1 Mitsubishi robot.

\section{REFERENCES}

[1] Ricard, R. and Gosellin, C. M., 1998, "On the Determination of the Workspace of Complex Planar Robotic Manipulators", ASME Journal of Mechanical Design, Vol.120, pp.269-278.

[2] Ceccarelli, M., 1995, “A Synthesis Algorithm for Three-Revolute Manipulators by Using an Algebraic Formulation of Workspace Boundary", Transactions of the ASME, Vol.117, pp.298-302.

[3] Snyman, J. A. and Plessis, L., 2000, "An optimization Approach to the Determination of the Boundaries of Manipulator Workspaces", ASME Journal of Mechanical Design, Vol.122, pp.447-456.

[4] Yang, J., Yu, W., Kim, J., and Malek, K.A., 2009, " On the placement of open-loop robotic manipulators for reachability", Journal of Mechanism and Machine Theory, 44(4), pp 671-684.

[5] Kumar, K. and Waldron, 1981, "The Workspace of a Mechanical Manipulator", ASME Journal of Mechanical Design, Vol.103, pp.665-672.

[6] Bi, Z. M., Lang, S.Y.T., 2009, "Joint workspace of parallel kinematic machines", Journal of Robotics and Computer Integrated Manufacturing, 25(1), pp 57-63.

[7] Malek and Yeh, 2000, "On the Placement of Serial Manipulators", Proceedings of DETC00, 2000, ASME Design Engineering Technical Conferences.

[8] Malek and Yeh, 2000, "Interior and Exterior Boundaries to the Workspace of Mechanical Manipulator", Journal of Robotics and Computer Integrated Manufacturing, Vol.16, pp.365-376.

[9] Cao, Y., Qi, S., Lu, K., Zang, Y., and Yang, G., 2009, "An integrated method for workspace computation of robot manipulator", Proceedings of the International Joint conference on Computational Sciences and Optimization, pp 309-312.

[10] Jo, D. Y. and Haug, E. J., 1989, "Workspace Analysis of Multibody Mechanical Systems Using Continuation Methods", Journal of Mechanisms, Transmissions, and Automation in Design, Vol.111, pp.581-58.

[11] Pitarch, E., Yang, J., and Malek, A.K., 2005, "Santos Hand: Workspace Analysis", Proceedings of International conference on Modeling and Simulation, Cancun, Maxico.

[12] Malek, K., 1997, "On Determination of the Boundaries to the Workspace of the Manipulator", Journal of Robotics and Computer Integrated Manufacturing, Vol.13, No.1, pp.63-72.

[13] Tsai, T. and Soni, A., 1983, "An Algorithm for the Workspace of a General n-R Robot", ASME Journal of Mechanical Design, Vol.105, pp.52-57.

[14] Shaheen, A. and Luo, S., 1998, "Analysis of Kinematic singularities for Robot Manipulators in Cartesian Coordinate Parameters", IEEE International Conference on Robotics and Automation.

[15] Malek, K. and Yang, J., 2000, "Method and Code for the Visualization of Multivariate Solids", Proceedings of DET00, 2000 ASME Design Engineering Technical Conferences.

J oumal of Mechanical Engineening, Vol. ME 41, No. 1, J une 2010 Transaction of the Mech. Eng. Div., The Institution of Eng ineers, Bangladesh 